\title{
Generation of fuzzy edge images using trapezoidal membership functions
}

\author{
C. Lopez-Molina ${ }^{1}$ H. Bustince ${ }^{1}$ J. Fernandez ${ }^{1}$ B. De Baets ${ }^{2}$ \\ ${ }^{1}$ Dpto. Automática y Computación, Universidad Pública de Navarra, Spain \\ ${ }^{2}$ Dept. of Applied Mathematics, Biometrics and Process Control, Ghent University, Belgium
}

\begin{abstract}
The fuzzy representation of the edges has been widely studied in different works. Generally, for each pixel, the authors use membership degrees linearly proportional to the magnitude of the gradient at that position of the image. This would be equivalent to using a triangular membership functions on the gradient magnitude. In this work we study the use of parametric functions in the transformation of the gradient images into fuzzy sets. The results obtained with different parametrical functions are studied using Baddeley's Delta Metric.
\end{abstract}

Keywords: Image processing, Edge detection, Fuzzy edges, Membership functions

\section{Introduction}

There exists in the literature a wide variety of techniques for edge detection. Most of them face the problem as a single task, not defining clear borders between the different processing steps. However, Bezdek et al. [1] introduced a breakdown structure in four different phases. In this structure each of the phases is determined naturally by the meaning of the information it handles.

Initially we start with a grayscale image. After conditioning ( $1^{\text {st }}$ phase), we have an image prepared for a better edge detection. Examples of this procedure are denoising or contrast enhancing. The feature extraction ( $2^{\text {nd }}$ phase) associates with each pixel a given number of values, representing the characteristics of the intensity changes around its positions. Some operators for feature extraction are, for example, Sobel operators [2]. Then, blending ( $3^{\text {rd }}$ phase) consists of combining the different features obtained in the previous phase into a single value, typically known as edginess. To finish, scaling ( $4^{\text {th }}$ phase) turns the edginess image into the appropriate representation format. This format is typically binary, thin edges, after Canny constraints [3].

This structure offers great flexibility, but at the same time adjusts to different strategies for edge detection. However, it is the phase of blending the one that usually takes less attention in the literature. This fact is specially noteworthy in the gradientbased edge detection methods $([3,4,5])$, where the magnitude of the gradient is taken as edginess, being the Euclidean norm the most common choice
[6]. That is, a membership degree linearly proportional to the magnitude of the gradient is considered. However, the fuzzy membership does not need to be linearly proportional to the measurable fact it is derived from. In this work we propose changing this relationship of linearity between the gradient at a pixel and the membership degrees to the edges. In order to generate membership degrees, we use a simple parametric model generalizing the triangular function in the traditional approach. Then, we study the combinations of parameters and its results in the processing of the edges in natural images.

The remainder of the work is organized as follows. Section 2 analyzes different fuzzy approaches to edge detection. In Section 3 we introduce the mathematical foundations of the parametric model. Then, some experiments are included in Section 4. We finish with some brief conclusions.

\section{On the fuzzy representation of the edges in images}

A digital image is a discrete representation of the reality, and carries some implicit ambiguity. This ambiguity manifests in two different facts: the color of the pixels (since there is a wide, yet limited number of tones available) and the position of the objects (because of the discrete number of pixels). In addition, there are several other problems in the information analysis, such as the noise.

Any possible treatment of the image will have to account for the problems associated with the discretization of the image. For example, sometimes it is impossible to discern which object does one pixel belong to. Therefore, even a human has some uncertainty about the true position of the edges on an image. Considering this, the fuzzy logic appears as a convenient choice for handling the intermediate representation of the edges $[7,8]$.

Many different strategies have been undertaken for applying the fuzzy logic in the edge detection problem. Some examples are the treatment of the image as an interval-valued fuzzy set [9], the gravitational approach [10], the fuzzy morphology $[11,12]$ or the rule-based systems $[1,13,14,15]$. All these methods, at some point of the procedure, represent the edges of the image in a fuzzy way. That is, they generate an image where a value is associated with a position, representing the mem- 

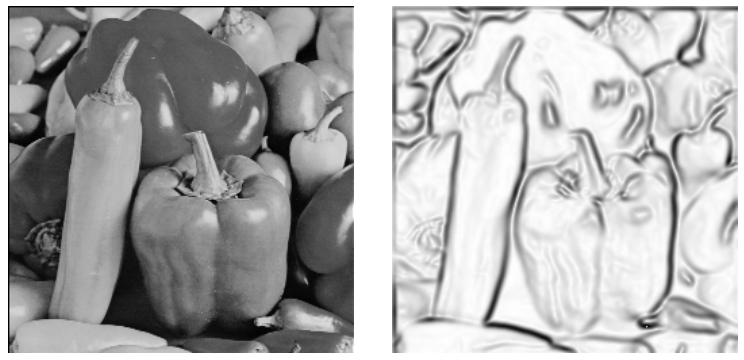

Figure 1: Peppers image (left) and a fuzzy representation of its edges (right).

bership degree to the edges fuzzy set. Fig. 1 contains one example of Peppers image along with one of such representations of the edges. We can observe how the representation covers both dimensions of the uncertainty in the image: the memberships to the edges vary gradually (representing the tonal ambiguity) and the edges spread along several positions (assuming the spatial ambiguity).

However, this idea collides with the restrictions stated by Canny $[16,17,3]$. These restrictions force the edges to be represented as binary images with edge lines being no wider than 1 pixel. Some examples of the techniques used in this transformation are $\alpha$-cuts [18], non-maximum suppression [3] hysteresis [19] or distance-based defuzzifications $[15,20]$. Given the complexity of the transformation of the fuzzy images into binary images (happening at the scaling phase), some authors use both representations for illustrating the performance of a detector [15].

In this work we analyze the impact of the shape of the fuzzy set used in the conversion of gradient magnitudes into membership degrees.

\section{Using membership functions}

Considering the breakdown structure by Bezdek et al., this work is centered in the so-called blending phase. Specifically, we analyze and model the relationship between the gradient used to characterize the intensity changes around a pixel and the membership degree to the edge it is assigned. That is, we focus on the relationship between the measurable fact (occurrence of different intensities in adjacent pixels) and its fuzzy representation. This step has usually had few or no consideration in the literature. In a vast majority of the cases, the magnitude of the gradient, once normalized, is taken as a membership degree. However, the fuzzy set generated using this strategy is meaningless; That is, it is a set constructed straight from data, without semantics or interpretation of any kind.

One interesting proposal for mapping gradients to membership degrees introducing interpretation was presented by Bezdek et al. [1]. The authors propose a Takagi-Sugeno (T-S) model with the pixel gradient components as inputs, and the fuzzy mem-

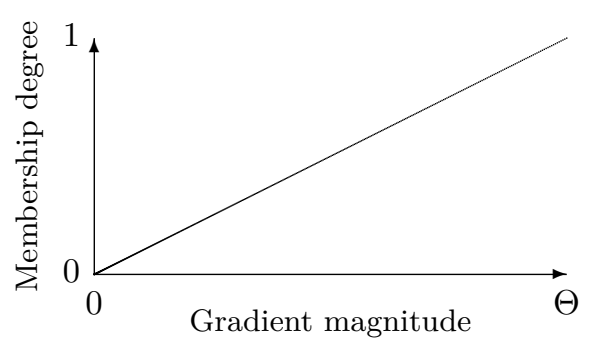

(a) Traditional membership function

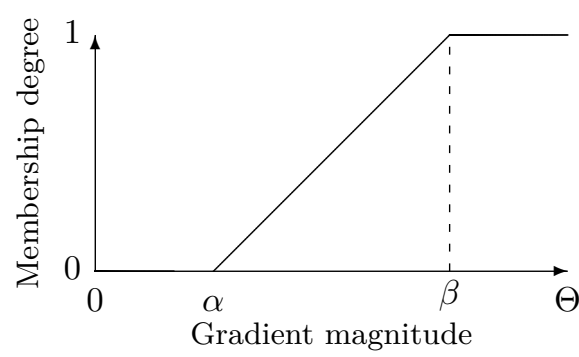

(b) Proposed membership function

Figure 2: Membership functions for converting gradient magnitudes into membership degrees.

bership as output. This idea is very interesting, but demands the use of training techniques. In case the model is not trained, we find a static, straight mapping of the gradient magnitudes into fuzzy membership degrees. Also Law et al. [21], Liang et al. [13] or Russo [22] did interesting uses of fuzzy logic for blending, using rules. Nevertheless, edge detection methods do not usually perform well with training models, since the same pixel neighborhoods might or might not be considered as edges, depending on the context of the image. Hence, the generation of training data is complicated, unless we seek an edge detector specially adapted for some specific kind of edges.

It is noteworthy to mention at this point the fact that other detectors (specifically those based on patterns and rules $[14,15,23])$ generate edginess values in a different way (generally using inference models). That is, even if they map the gradients to membership degrees using triangular functions, they combine more information to obtain edginess values. In these cases the relationship between the happening (intensity change) and the fuzzy representation might or might not be linear, usually depending on the inference models. In this work we focus on the methods based on gradients, that usually have more impact in the literature

The traditional idea is the one observed in Fig. 2(a), where $\Theta$ represents the greater magnitude a gradient can have on an image. Intuitively we understand that, the larger the gradient, the greater the membership degree to the edges. However, this fact only justifies the monotonicity, which can be 


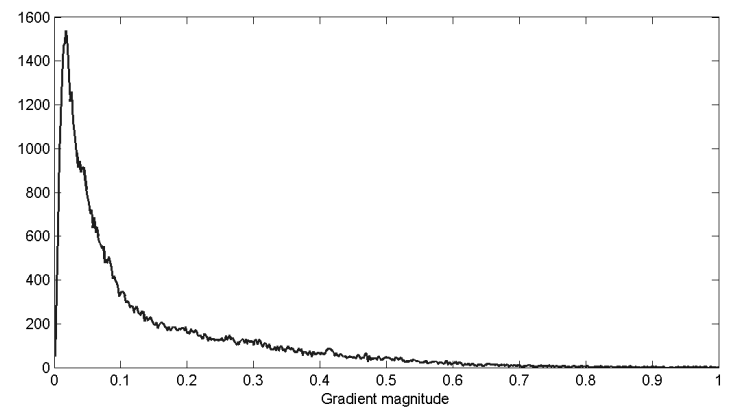

Figure 3: Histogram of magnitudes of the gradients in the Peppers Image (Fig. 1).

given in many different shapes. We propose a simple generalization of the traditional approach, such as the one in Fig. 2(b). This model assumes that the edges at some positions are small enough for them to be discarded as true edges, while some other are large enough to be directly considered as edges. The only restriction applying on the model is $\alpha \leq \beta$. The interpretation of the model is simple. The parameter $\alpha$ represents the minimal magnitude a gradient must have to be considered as an edge. On the other hand, we have $\beta$ being the threshold after which any gradient is considered to be produced by a true edge. An special case of the model happens when $(\alpha, \beta)=(0,1)$. This situation is equivalent to the traditional model. Alternatively, any case satisfying $\alpha=\beta$ is equivalent to crisp thresholding.

In order to fully understand the model we have to analyze the gradients in an edge image. Generally the histogram of gradient magnitudes (HGM) of an image is unimodal, being most of the pixels in the lower side $[24,18]$. That is, most of the pixels are assigned relatively small gradients. We have to keep in mind that the gradient on a pixel can not be calculated, since the image is a discrete environment (see [25] or [26] for considerations on the discrete/continuous duality in image processing). Hence, different ways of estimating the gradient can lead to different results. However, the resulting HGM will always be unimodal. Some deeper considerations on the search for gradients in digital images can be found in $[26,27,28]$.

Fig. 3 includes the HGM of the Peppers image in Fig. 1. In this case, where the gradients have been obtained using normalized Sobel operators [2], we can observe that most of the gradients usually have small magnitudes. In this specific example the average gradient is 0.135 , while $40 \%$ of the pixels are assigned gradients with a magnitude under 0.05. Hence, when using our models, we will have to choose relatively low values for the parameter $\alpha$, since larger ones would discard a large number of pixels. Alternatively, small variations of the parameter $\beta$ should have smalled significance, as they would affect to a limited number of pixels.

The fuzzy representation of the edges in an image

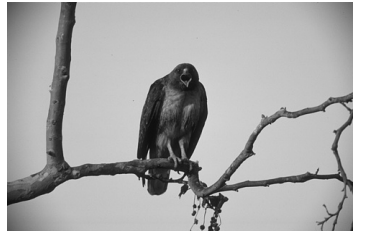

Original image

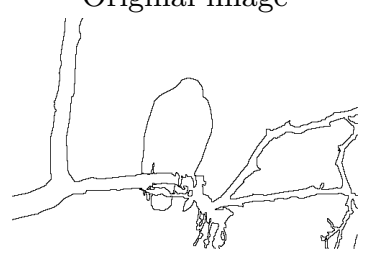

Hand-made solution 2

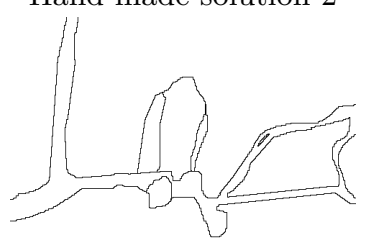

Hand-made solution 4

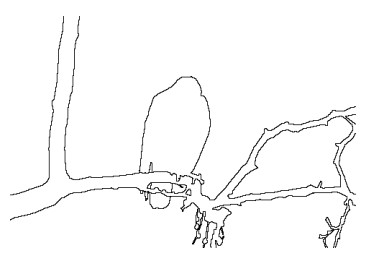

Hand-made solution 1

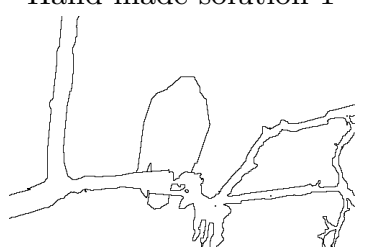

Consensus solution 3

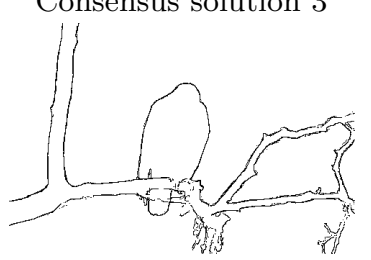

Consensus solution
Figure 5: Image 42092 of the BSDS test set, along with 4 of its hand-made solutions and the consensus one.

is the intermediate step before binarization. Very often the binarization is usually carried out in an supervised way [3], being an exception some of the methods based on single [24, 18] or double thresholds $[29,19,30]$. By using these unsupervised methods the impact of the adjustments of $\alpha$ and $\beta$ are hard to predict. Typically, large values of $\alpha$ will discard many points (producing false negatives) while small values of $\beta$ will force many pixels to be considered edges (producing false positives). However, it is complicated to predict the role of the slope in the interval $[\alpha, \beta]$ in the final edges.

Some examples of the generation of the fuzzy set in the Peppers image are illustrated in Fig. 4. The results after binarization, using the technique in [19], are displayed as well.

\section{Experimental test}

\subsection{Test data}

In the experiment we have used the Berkeley Segmentation Dataset (BSDS) [31]. This dataset contains natural images, each of them provided with 5 to 10 hand-made solutions, in the shape of binary edge images. We have used the first 40 images of the test set. All of the images are provided in grayscale with $321 \times 481$ (or $481 \times 321$ ) pixels resolution.

All of the hand-made solutions associated with one image have been combined in order to create a single solution. In order to do so, we have used the consensus generation techniques by FernándezGarcía et al. [32]. Specifically, we have used the minimean version of the technique. An example of this process is illustrated in Fig. 5, where we include 


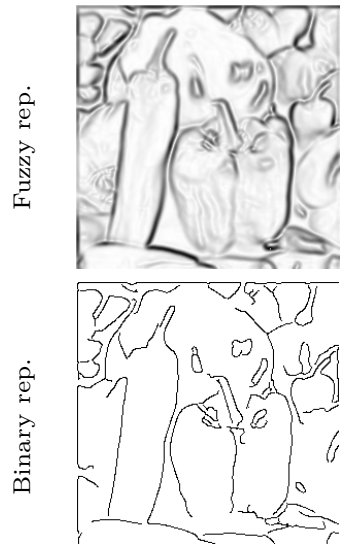

(a) $\alpha=0, \beta=1$

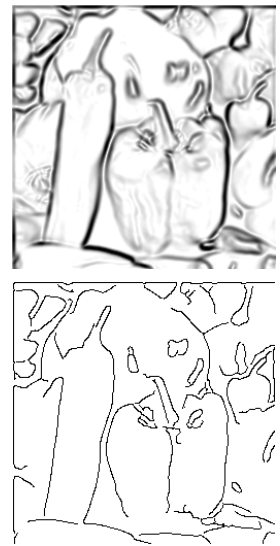

(b) $\alpha=0.05, \beta=0.8$

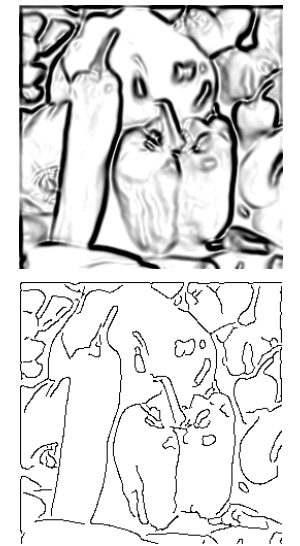

(c) $\alpha=0.05, \beta=0.5$

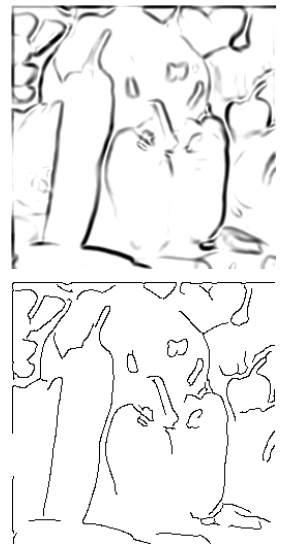

(d) $\alpha=0.20, \beta=0.8$

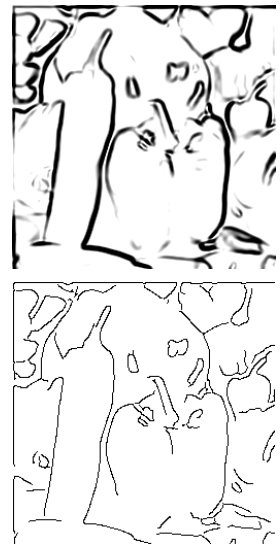

(e) $\alpha=0.20, \beta=0.5$

Figure 4: Fuzzy and crisp edges generated from Peppers image after using different value for the parameters $\alpha$ and $\beta$. Note that $\alpha=0, \beta=1$ recovers the approach with a triangular function.

an original image, 4 of the hand-made solutions provided in the BSDS and the consensus image generated from them.

\subsection{Comparison method}

Baddeley's Delta Metric (BDM) has been selected for the comparison of the binary results. This metric, inspired by Hausdorff distance, was originally designed for the comparison of binary sets. However, it can also be applied on the comparison of binary images, since these images can be considered as subset of points in a discrete referential.

Let $I_{1}$ and $I_{2}$ be two binary images with $M$ rows and $N$ columns. We consider the set of their positions to be $P=\{1, \ldots, M\} \times\{1, \ldots, N\}$. The edges of each image are two subsets of points $B_{1}$ and $B_{2}$ such that $B_{i}=\left\{p \in P \mid I_{i}(p)=1\right\}$. The distance between $I_{1}$ and $I_{2}$, denoted $\Delta_{w}^{k}\left(I_{1}, I_{2}\right)$ is defined as

$$
\Delta_{w}^{k}\left(I_{1}, I_{2}\right)=\left[\frac{1}{|P|} \sum_{p \in P}\left|w\left(d\left(p, B_{1}\right)\right)-w\left(d\left(p, B_{2}\right)\right)\right|^{k}\right]^{\frac{1}{k}}
$$

where $1<k<\infty$ and $d\left(p, B_{i}\right)$ is the distance between position $p$ and the closest position $p^{\prime} \in P$ satisfying $B_{i}\left(p^{\prime}\right)=1$. To finish, $w: \mathbb{R} \rightarrow \mathbb{R}$ can be any concave function. In this experiment, as in [24, 19], we will consider $k=2$, the Euclidean distance will be taken as $d$, and $w(x)=x$.

\subsection{Experimental procedure}

The experimental procedure, structured as established by Bezdek et al. in [1], is as follows

(A) Conditioning-Smoothen the image with a regular Gaussian filter generated with $\sigma=1.0$ [16]. More information about the effect of regular Gaussian smoothing (also known as Gaussian scale-space) can be found in [27, 28].

(B) Feature extraction- Extract the directional components of the gradient $\left(f_{x}, f_{y}\right)$ using the operators by Canny [3, 16], using $\sigma_{2}=1.80$. This value is common, considering the size of the images. Different studies on the edge extraction operators and their scale factors have been presented by Canny [3], Heath et al. [33] and Lindeberg [27].

(C) Blending-Calculate the magnitude of the gradient using the Euclidean norm $d_{2}$. Then, using a pair $(\alpha, \beta)$, create a function to associate each element of the image a membership degree. That is, considering directional components of the gradient $f_{x}$ and $f_{y}$, we have that the edginess of a pixel $p$ is

$$
B_{\alpha, \beta}(p)= \begin{cases}0 & , \text { if } \frac{d_{2}\left(f_{x}, f_{y}\right)}{\sqrt{2}}<\alpha \\ \frac{d_{2}\left(f_{x}, f_{y}\right)-\alpha}{\beta-\alpha} & , \text { if } \alpha \leq \frac{d_{2}\left(f_{x}, f_{y}\right)}{\sqrt{2}}<\beta \\ 1 & \text { otherwise }\end{cases}
$$

To finish, we have applied non-maximum suppression (NMS) technique, as introduced by Canny [3]. This technique allows to thin the edges when the transition between 2 objects is spread over several positions. Still, edges keep fuzzy memberships to the edges.

(D) Scaling- We have used hysteresis for binarizing the edges. This technique allows to select which intensity changes are relevant enough to be selected as edges, and which are to be ignored, based on their membership degrees. In this process, we use two different thresholds $T_{1}, T_{2} \in[0,1]$, with $T_{1}<T_{2}$. All of the pixels having a membership degree over $T_{2}$ will be automatically considered as edges, while those whose membership degree is under $T_{1}$ are discarded. Then, the pixels with a membership degree between the thresholds are selected if and only if they are connected to other pixels with a value over $T_{1}$. In order to determine the values of these thresholds we have used the unsupervised technique by Medina-Carnicer et al. [24]. 


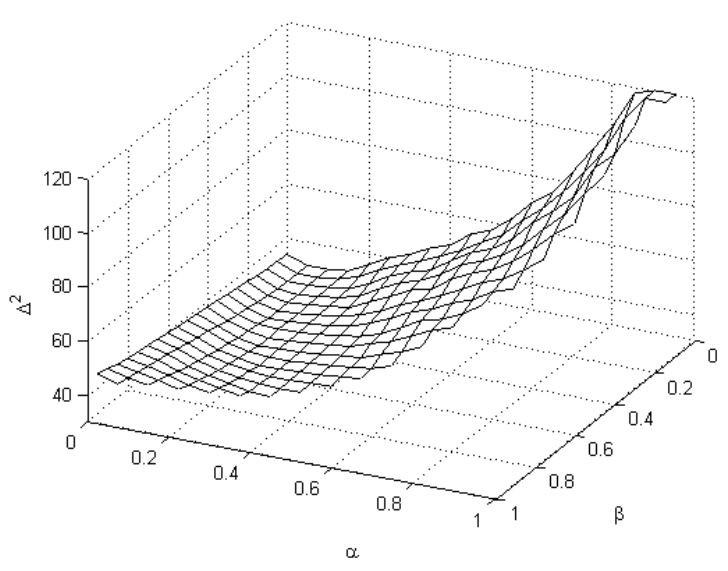

Figure 6: Average DBM in the dataset using different $\alpha$ and $\beta$ values.

A different choice of the parameters might result in different performance. However, the purpose of this experiment is to compare different ways of assigning the membership degrees to the pixels. That is, comparing the different $\alpha$ and $\beta$ parameterizations, including the classical $(\alpha, \beta)=(0,1)$, in some fixed conditions.

\subsection{Results}

The tests have been run with all the combinations of parameters $\alpha, \beta \in\{0,0.05, \ldots, 0.95,1\}$ satisfying $\alpha \leq \beta$. The average $\mathrm{BDM}$ for each pair $(\alpha, \beta)$ is illustrated in Fig. 6 . In the Table 1 we have detailed the results obtained with $\alpha \in[0,0.45]$. In this table we display the difference of the performance of each pair of parameters and the performance of the best possible choice. In this case, the best possible choice is $\alpha=0.1$ and $\beta=0.25$, scoring an average BDM of (30.63). To finish, in Fig. 7 we include a visual distribution of the best results obtained in the experiment.

In Fig. 6 we can observe how the best results (lower distances to the solutions) correspond to low $\alpha$ values. This reflects the fact that, if the value of $\alpha$ is high, most of the pixels obtain a membership degree 0 , and are hence discarded. We also observe how, once the value of $\alpha$ is fixed, the relevance of the choice of $\beta$ is low.

In Table 1 and Fig. 7 , the first thing to be noticed is the fact that many combinations of $\alpha$ and $\beta$ perform better than the original normalization approach (represented by $\alpha=0$ and $\beta=0$ ). This indicates that the performance of the Canny method can be improved by using a trapezoidal function in the generation of a fuzzy edges image from a gradients image. We also observe how the best results spread around a coordinate increase of $\alpha$ and $\beta$. In fact, the best results are with combinations satisfying $1.5 \alpha \leq \beta \leq 3 \alpha$. In this way, we find combinations of parameters as $(\alpha, \beta)=(0.3,0.7)$ (equivalent to an $\alpha$-cut of the fuzzy set) generating very com-
() Best result

Diff. to the best result $<0.5$

Diff. to the best result $<1$

Diff. to the best result $<2$

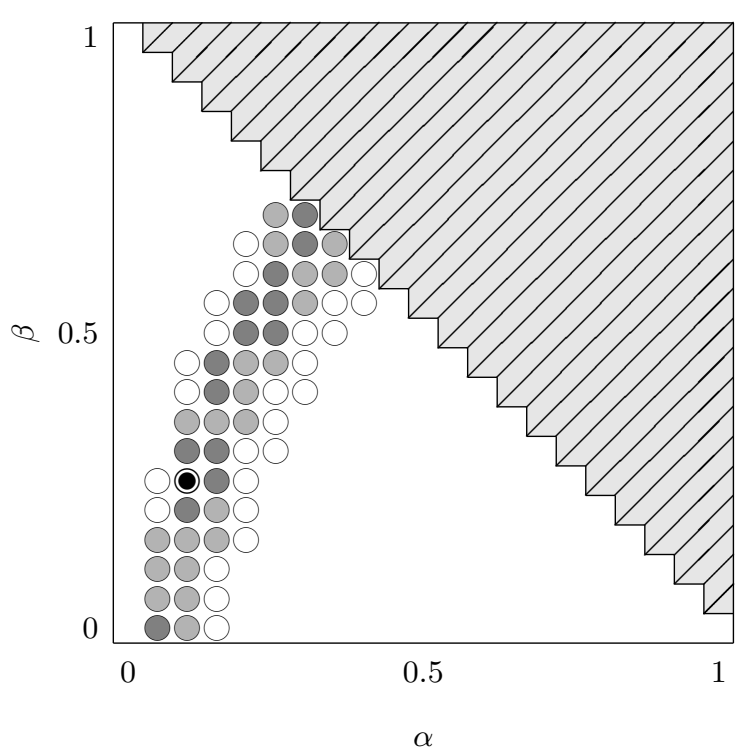

Figure 7: Distribution of the combination of the parameters $\alpha$ and $\beta$ producing the most competitive results.

petitive results.

\section{Conclusions}

We have analyzed the generation of membership degrees to the edges from the magnitude of the gradients associated with each pixel. Specifically, we have considered the use of trapezoidal membership functions in the conversion of the gradients into membership degrees. We have presented a simple model, based in 2 parameters determining the trapezoidal shape of the membership function. This model is a generalization of the classical approximation, where the membership degree is linearly proportional to the magnitude of the gradient. To finish, we have shown experimentally how the adoption of those models can improve the results of the Canny edge detection method.

\section{Acknowledgements}

This work has been partially funded by the Spanish Ministry of Science, project TIN2010-15055, and by the Research Services of the Public University of Navarra.

\section{References}

[1] J.C. Bezdek, R. Chandrasekhar, and Y. Attikouzel. A geometric approach to edge detec- 


\begin{tabular}{|c|c|c|c|c|c|c|c|c|c|c|c|}
\hline & \multicolumn{10}{|c|}{$\alpha$} \\
\hline & & 0.00 & 0.05 & 0.10 & 0.15 & 0.20 & 0.25 & 0.30 & 0.35 & 0.40 & 0.45 \\
\hline \multirow{21}{*}{$\infty$} & 0.00 & +3.4 & +0.7 & +0.7 & +1.6 & +5.3 & +9.3 & +11.9 & +15.7 & +18.3 & +23.7 \\
\hline & 0.05 & +3.7 & +0.6 & +0.6 & +1.5 & +3.8 & +7.7 & +10.9 & +13.7 & +17.1 & +20.6 \\
\hline & 0.10 & +3.8 & +0.7 & +0.7 & +1.3 & +2.3 & +5.5 & +9.8 & +12.7 & +14.8 & +19.7 \\
\hline & 0.15 & +4.2 & +0.9 & +0.1 & +1.0 & +1.9 & +3.9 & +8.0 & +11.6 & +14.0 & +17.6 \\
\hline & 0.2 & +5.1 & +1.4 & +0.2 & +0.8 & +1.7 & +2.6 & +6.1 & +9.5 & +13.3 & +14.9 \\
\hline & 0.25 & +5.5 & +1.8 & $(30.6)$ & +0.4 & +1.3 & +2.2 & +4.3 & +7.0 & +10.8 & +13.7 \\
\hline & 0.3 & +6.1 & +2.6 & +0.1 & +0.5 & +1.1 & +1.7 & +3.0 & +5.6 & +8.7 & +12.5 \\
\hline & 0.35 & +6.8 & +3.0 & +1.0 & +0.6 & +0.7 & +1.4 & +2.4 & +3.6 & $\begin{array}{r}+5.9 \\
\end{array}$ & $\begin{array}{r}+9.6 \\
\end{array}$ \\
\hline & 0.4 & +7.4 & +3.7 & +1.3 & +0.1 & +0.6 & +1.2 & +1.9 & +2.8 & +5.3 & +7.4 \\
\hline & 0.45 & +7.7 & +4.8 & +1.7 & +0.2 & +0.6 & +0.8 & +1.4 & +2.3 & +2.9 & +5.6 \\
\hline & 0.5 & +8.2 & +5.8 & +2.7 & +1.0 & +0.4 & +0.3 & +1.1 & +1.6 & +2.3 & +3.3 \\
\hline & 0.55 & +8.7 & +6.3 & +3.1 & +1.5 & +0.1 & +0.4 & +0.7 & +1.0 & +1.8 & +2.4 \\
\hline & 0.60 & +9.4 & +7.1 & +4.0 & +2.4 & +1.2 & +0.4 & +0.6 & +0.9 & +1.1 & - \\
\hline & 0.65 & +10.0 & +8.0 & +5.0 & +2.8 & +1.5 & +0.8 & +0.3 & +0.5 & - & - \\
\hline & 0.70 & +10.7 & +8.9 & +5.9 & +3.9 & +2.4 & +0.8 & +0.3 & $\begin{array}{ll}- \\
-\end{array}$ & - & - \\
\hline & 0.75 & +11.1 & +9.6 & +7.0 & +5.1 & +3.5 & +2.3 & - & - & - & - \\
\hline & 0.80 & +11.7 & +10.3 & +8.4 & +6.2 & +4.8 & - & - & - & - & - \\
\hline & 0.85 & +12.5 & +11.0 & +9.4 & +7.5 & - & - & - & - & - & - \\
\hline & 0.90 & +14.0 & +11.8 & +10.5 & - & - & - & - & - & - & - \\
\hline & 0.95 & +14.8 & +12.5 & - & - & - & - & - & - & - & - \\
\hline & 1.00 & +17.8 & - & - & - & - & - & - & - & - & - \\
\hline
\end{tabular}

Table 1: Difference of performance between each pair $(\alpha, \beta)$ and the best possible solution in the experiment, (30.63, obtained with $\alpha=0.1$ and $\beta=0.25)$.

tion. IEEE Trans. on Fuzzy Systems, 6(1):5275,1998

[2] I. Sobel and G. Feldman. A 3x3 isotropic gradient operator for image processing. Presented at a talk at the Stanford Artificial Project, 1968.

[3] J. Canny. A computational approach to edge detection. IEEE Trans. on Pattern Analysis and Machine Intelligence, 8(6):679-698, 1986.

[4] Genyun Sun, Qinhuo Liu, Qiang Liu, Changyuan Ji, and Xiaowen Li. A novel approach for edge detection based on the theory of universal gravity. Pattern Recognition, 40(10):2766-2775, 2007.

[5] C. Lopez-Molina, H. Bustince, J. Fernandez, P. Couto, and B. De Baets. A gravitational approach to edge detection based on triangular norms. Pattern Recognition, 43(11):3730-3741, 2010.

[6] C. Lopez-Molina, J. Fernandez, A. Jurio, M. Galar, M. Pagola, and B. De Baets. On the use of quasi-arithmetic means for the generation of edge detection blending functions. In Proceedings of the IEEE International Conference on Fuzzy Systems, 2010.

[7] E. Kerre and M. Nachtegael, editors. Fuzzy techniques in Image Processing. Physica-Verlag HD, 2000.

[8] M. Nachtegael, D. van der Weken, E. Kerre, and W. Philips, editors. Soft Computing in Image Processing: Recent Advances. Springer, 2006.

[9] H. Bustince, E. Barrenechea, M. Pagola, and J. Fernandez. Interval-valued fuzzy sets constructed from matrices: Application to edge detection. Fuzzy Sets and Systems,
160(13):1819-1840, 2009.

[10] C. Lopez-Molina, Humberto Bustince, J. Fernández, Edurne Barrenechea, Pedro Couto, and Bernard De Baets. A t-norm based approach to edge detection. In IWANN Proceedings (1), Lecture Notes in Computer Science, pages 302-309. Springer, 2009.

[11] B. De Baets, E. Kerre, and M. Gupta. The fundamentals of fuzzy mathematical morphology. Part 1: Basic concepts. Internat. J. General Systems, (23):155-171, 1994.

[12] Yuqian Zhao, Weihua Gui, and Zhencheng Chen. Edge detection based on multi-structure elements morphology. Intelligent Control and Automation, 2006. WCICA 2006. The Sixth World Congress on, 2:9795-9798, June 2006.

[13] Lily Rui Liang and Carl G. Looney. Competitive fuzzy edge detection. Applied Soft Computing, 3(2):123-137, 2003.

[14] F. Russo and G. Ramponi. Edge extraction by fire operators. In Proceedings of the Third IEEE Conference on Fuzzy Systems, IEEE World Congress on Computational Intelligence, volume 1, pages 249-253, Jun 1994.

[15] Liming $\mathrm{Hu}, \mathrm{H} . \mathrm{D}$. Cheng, and Ming Zhang. A high performance edge detector based on fuzzy inference rules. Information Sciences, 177(21):4768-4784, 2007.

[16] M. Basu. Gaussian-based edge-detection methods-a survey. IEEE Trans. on Systems, Man, and Cybernetics, Part C: Applications and Reviews, 32(3):252-260, 2002.

[17] J. Canny. Finding edges and lines in images. Technical report, Massachussets Institute of Technology, Cambridge, MA, USA, 1983. 
[18] Paul L. Rosin. Unimodal thresholding. Pattern Recognition, 34(11):2083-2096, 2001.

[19] R. Medina-Carnicer, F.J. Madrid-Cuevas, A. Carmona-Poyato, and R. Muñoz-Salinas. On candidates selection for hysteresis thresholds in edge detection. Pattern Recognition, 42(7):1284-1296, 2009.

[20] Natasa Sladoje, Joakim Lindblad, and Ingela Nyström. Defuzzification of spatial fuzzy sets by feature distance minimization. Image and Vision Computing, 29(2-3):127-141, 2011.

[21] Todd Law, Hidenori Itoh, and Hirohisa Seki. Image filtering, edge detection, and edge tracing using fuzzy reasoning. IEEE Trans. on Pattern Analysis and Machine Intelligence, 18(5):481-491, 1996.

[22] Fabrizio Russo. Fire operators for image processing. Fuzzy Sets and Systems, 103(2):265275, 1999.

[23] Dong-Su Kim, Wang-Heon Lee, and In-So Kweon. Automatic edge detection using $3 \mathrm{x}$ 3 ideal binary pixel patterns and fuzzy-based edge thresholding. Pattern Recognition Letters, 25(1):101-106, 2004.

[24] R. Medina-Carnicer and F.J. Madrid-Cuevas. Unimodal thresholding for edge detection. Pattern Recognition, 41(7):2337-2346, 2008.

[25] Robert M. Haralick. Digital step edges from zero crossing of second directional derivatives. IEEE Trans. on Pattern Analysis and Machine Intelligence, 6(1):58-68, 1984.

[26] V. Torre and T. Poggio. On edge detection. IEEE Trans. on Pattern Analysis and Machine Intelligence, 8:147-163, 1984.

[27] Tony Lindeberg. Edge detection and ridge detection with automatic scale selection. International Journal of Computer Vision, 30(2):117-
$156,1998$.

[28] J. Weickert, B.M.T.H. Romeny, and M.A. Viergever. Efficient and reliable schemes for nonlinear diffusion filtering. IEEE Trans. on Image Processing, 7(3):398-410, 1998.

[29] R. Medina-Carnicer, A. Carmona-Poyato, R. Muñoz-Salinas, and F. J. Madrid-Cuevas. Determining hysteresis thresholds for edge detection by combining the advantages and disadvantages of thresholding methods. IEEE Trans. on Image Processing, 19(1):165-173, 2010.

[30] Y. Yitzhaky and E. Peli. A method for objective edge detection evaluation and detector parameter selection. IEEE Trans. on Pattern Analysis and Machine Intelligence, 25(8):10271033, 2003.

[31] D. Martin, C. Fowlkes, D. Tal, and J. Malik. A database of human segmented natural images and its application to evaluating segmentation algorithms and measuring ecological statistics. In Proceedings of the 8th International Conference on Computer Vision, volume 2, pages 416-423, 2001.

[32] N.L. Fernández-García, A. Carmona-Poyato, R. Medina-Carnicer, and F.J. Madrid-Cuevas. Automatic generation of consensus ground truth for the comparison of edge detection techniques. Image and Vision Computing, 26(4):496-511, 2008.

[33] M.D. Heath, S. Sarkar, T. Sanocki, and K.W. Bowyer. A robust visual method for assessing the relative performance of edge-detection algorithms. IEEE Trans. on Pattern Analysis and Machine Intelligence, 19(12):1338-1359, 1997. 